\title{
Metabolomics and Proteomics Reveal the Variation of Substances in Apheresis Platelets during Storage and Their Effects on Cancer Cell Proliferation
}

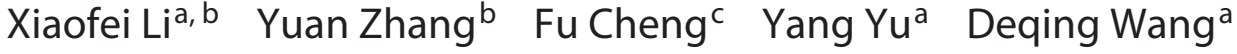 \\ a Department of Blood Transfusion, Chinese PLA General Hospital, Beijing, China; \\ ${ }^{b}$ Department of Blood Transfusion, Beijing Friendship Hospital, Capital Medical University, Beijing, China; \\ 'Department of Blood Transfusion, West China Hospital of Sichuan University, Chengdu, China
}

\section{Keywords}

Apheresis platelets · Metabolites · Exosomes · Cancer cells
AP supernatants may inhibit the proliferation of cancer cells but the variant terminal AP exosomes have no effect on cancer cell proliferation.

(c) 2020 S. Karger AG, Basel

\begin{abstract}
Introduction: Apheresis platelets (APs) are clinically and crucially important in the prevention and treatment of bleeding in patients with thrombocytopenia or cancer. However, few researchers have addressed the variation of supernatant metabolites and exosome proteins in APs during storage and their effects on cancer cell proliferation. Objective: This study was designed to explore the change rules of the metabolites and exosomal proteins of APs during storage and their effects on cancer cell proliferation. Methods: Metabolomics and proteomics were separately applied to analyze the variation of AP supernatant metabolites and exosomal proteins between freshly prepared day- 0 and day- 5 terminal-stored APs. Cell counting kit (CCK)-8 assay was performed to detect the effects of AP supernatants and exosomes on the proliferation of cancer cells. Results: We found that the supernatant metabolites and exosomal proteins in APs were significantly different on day 0 and day 5 , and that many differential metabolites and exosomal proteins were associated with cancer characteristics. Furthermore, the day5 AP supernatants had a greater inhibition of the proliferation of K562, HepG2, and HCT116 cancer cells, but the day- 5 AP exosomes had no significant effect on the proliferation of these cancer cells. Conclusion: The variant terminal-stored
\end{abstract}

\section{Introduction}

As a potential life-saving therapy, transfusion of apheresis platelets (APs) is routinely used in the treatment of thrombocytopenia-associated bleeding complications. Meanwhile, APs play an important role in the effective management of thrombocytopenia due to malignancy or chemotherapy-related complications, and they prevent hemorrhage in patients with hematological disorders after stem cell transplantation $[1,2]$.

However, in most areas including China, APs can only be stored for up to 5 days primarily due to the increased risk of bacterial contamination, although some studies have indicated that platelet concentrates can be stored for up to 7 days $[3,4]$. Furthermore, platelet storage lesions (PSLs) can occur during the storage of APs, accompanied by multiple changes including increased release of platelet extracellular vesicles (P-EVs), reduced activation in response to agonists, increased apoptosis and phosphatidylserine externalization, and secretion of platelet gran-

Xiaofei Li and Yuan Zhang contributed equally to this manuscript. 
ules [5]. These may affect platelet functional integrity and induce other side effects. Studies reveal that P-EVs, including platelet-derived exosomes $(30-100 \mathrm{~nm}$ in diameter) and platelet microvesicles $(100-1,000 \mathrm{~nm}$ in diameter) $[6,7]$, play a vital role in steering the disorders of the human immune system, such as reducing natural killer cell function [8] and inhibiting the IL-17 production of regulatory $\mathrm{T}$ cells [9]. Interestingly, there have been studies that indicate that platelet microvesicles, being part of $\mathrm{P}-\mathrm{EV}$, participate in the regulation of cancer $[10,11]$. All these studies suggest that the substance changes in AP solutions during storage may contribute to severe side effects of platelet transfusion; this requires more attention from clinicians and researchers.

There are studies that show the transcriptomic profiling of terminal platelets and P-EVs [12] and the variation of protein composition in human platelets during storage at different temperatures [13]. However, how substances change between freshly prepared day- 0 and day- 5 terminal-stored APs remains largely unknown. More importantly, the effects of the day- 0 and day- 5 AP supernatants and exosomes on the proliferation of cancer cells is yet unclear. To further investigate the variation of metabolites in AP supernatants and proteins in AP exosomes during storage, this study used metabolomics and proteomics to analyze, respectively, the variation of metabolites in stored AP supernatants and proteins in plateletderived exosomes between freshly prepared day-0 and day-5 terminal-stored APs. The effects of AP supernatants and exosomes on cancer cell proliferation were also detected.

\section{Materials and Methods}

\section{Collection of APs}

At the Chinese People's Liberation Army (PLA) General Hospital, APs were obtained from 10 healthy male blood donors, with a mean age of 33.8 years, according to the "Requirements for Health Examination of Blood Donors" (China, GB18467-2011).

\section{Isolation of AP Supernatants and Exosomes}

AP supernatants were obtained by centrifuging the day- 0 and day-5 stored APs, first at 2,000 $\mathrm{g}$ for $30 \mathrm{~min}$ at room temperature and then at $10,000 \mathrm{~g}$ for $30 \mathrm{~min}$ at $4{ }^{\circ} \mathrm{C}$. The AP supernatants were stored at $-80^{\circ} \mathrm{C}$ if not used. The AP supernatants were centrifuged at $105,000 \mathrm{~g}$ and $4{ }^{\circ} \mathrm{C}$ for $2 \mathrm{~h}$ to acquire primary AP exosomes (pellet) and exo-free-AP supernatants (supernatant). Subsequently, the primary exosomes extracted from $30 \mathrm{~mL}$ of AP supernatant were resuspended in $1 \mathrm{~mL}$ of PBS, and then filtered through a $0.22-\mu \mathrm{m}$-pore-size filter (Millipore; USA). After filtration, the primary AP exosomes were ultracentrifuged again at 105,000 $\mathrm{g}$ and $4^{\circ} \mathrm{C}$ for $2 \mathrm{~h}$ to get further purified exosomes. Finally, the AP exosomes were resuspended in $300 \mu \mathrm{L}$ of PBS and stored at $-80^{\circ} \mathrm{C}$. The AP exosomes were identified by means of Western blot and transmission electron microscopy (TEM) assays.

\section{AP Supernatant Metabolic Research Methods}

Extraction of Metabolites

All AP supernatant samples were thawed on ice, and then 300 $\mu \mathrm{L}$ of precooled extraction buffer (methyl-tert-butyl ether [MTBE]:methanol $=20: 6$ buffer) was mixed in $20 \mu \mathrm{L}$ of the samples. The mixture was subsequently vortexed for $2 \mathrm{~min}$, and then incubated at room temperature for $10 \mathrm{~min}$. The extraction mixture was then stored overnight at $-80^{\circ} \mathrm{C}$. After centrifugation of the extraction mixture at $12,000 \mathrm{~g}$ for $5 \mathrm{~min}$, the supernatant was transferred into new 96 -well plates and stored at $-80^{\circ} \mathrm{C}$ prior to performing liquid chromatography-mass spectrometry (LC-MS) analysis. In addition, quality control (QC) samples were also prepared by combining $10 \mu \mathrm{L}$ of each extraction mixture.

\section{LC-MS Analysis}

All samples were acquired by the LC-MS system according to the manufacturer's recommendations. First, all chromatographic separations were performed using an ultraperformance LC (UPLC) system (Shimazu, Japan). An ACQUITY UPLC C18 column (100 $\times 2.1 \mathrm{~mm}, 1.7 \mu \mathrm{m}$, Waters, UK) was used for the reversed phase separation. The column oven was maintained at $40^{\circ} \mathrm{C}$ with a flow rate of $0.3 \mathrm{~mL} / \mathrm{min}$, and the mobile phase consisted of solvent $\mathrm{A}$ (40\% water, $60 \% \mathrm{ACN}$, and $10 \mathrm{~mm}$ ammonium acetate) and solvent $\mathrm{B}$ ( $10 \% \mathrm{ACN}$ and $90 \% 2$-isopropanol). The gradient elution conditions were set as follows: $0-0.5 \mathrm{~min}, 20 \% \mathrm{~B}$; 0.5-1.5 min, 20 $40 \% \mathrm{~B} ; 1.5-10 \mathrm{~min}, 40-60 \% \mathrm{~B} ; 10-25 \mathrm{~min}, 60-100 \% \mathrm{~B} ; 25-26 \mathrm{~min}$, $100-20 \% \mathrm{~B} ; 26-30 \mathrm{~min}, 20 \% \mathrm{~B}$. The injection volume for each sample was $1 \mu \mathrm{L}$.

A high-resolution tandem mass spectrometer TripleTOF 6600 (SCIEX, UK) was used to detect metabolites eluted from the column. The Q-TOF was operated in both positive and negative ion modes. The curtain gas was set at 35 psi, ion source gas 1 at 60 psi, and ion source gas 2 at $60 \mathrm{psi}$; the interface heater temperature was $550^{\circ} \mathrm{C}$. The IonSpray Voltage Floating was set at $5,500 \mathrm{~V}$ in the positive ion mode and $-4,500 \mathrm{~V}$ in the negative ion mode. The MS data were acquired in the information dependent acquisition (IDA) mode. The TOF mass range was 240-1,250 Da. The survey scans were acquired in $250 \mathrm{~ms}$, and as many as 10 product ion scans were collected if a threshold of 100 counts/s was exceeded. Total cycle time was fixed at $1.3 \mathrm{~s}$. Four time-bins were summed for each scan at a pulser frequency value of $11 \mathrm{kHz}$ by monitoring the 40 $\mathrm{GHz}$ multichannel TDC detector with 4-anode/channel detection. During the acquisition, the mass accuracy was calibrated every 10 samples. To evaluate the stability of the LC-MS during the whole acquisition, a QC sample was acquired after every 10 samples.

\section{Analysis of Metabolomics Data}

The acquisition of MS raw data on pretreatments was performed using XCMS software. LC-MS raw data files were converted into the $m z \mathrm{XML}$ format and then processed by the XCMS, CAMERA, and metaX toolbox implemented with the $R$ software. The parameters of peak picking were set as follows: method: centWave; minfrac: 0.5 ; snthr: 6 ; ppm: 25; peakwidth: 5,25 ; bw2: 5 ; mzwid: 0.015 ; $m z$ diff: 0.01 ; profstep.OBIWarp: 0.1 . Each ion was identified by combining retention time (RT) and $m / z$ data. Intensities of each peaks were recorded and a 3-dimensional matrix containing arbitrarily assigned peak indices (retention time- $\mathrm{m} / \mathrm{z}$ pairs), sample names (observations), and ion intensity information (variables) was generated.

The online databases LIPID Metabolites and Pathways Strategy (LIPID MAPS), Kyoto Encyclopedia of Genes and Genomes (KEGG), and Human Metabolome database (HMDB) were used to annotate the primary metabolites by matching the exact molecular mass data $(\mathrm{m} / \mathrm{z})$ of samples with those from the database. If a mass difference between the observed and database value was 
$<10 \mathrm{ppm}$, the metabolite would be annotated, and the molecular formula of metabolites would further be identified and validated by the isotopic distribution measurements. We also used an inhouse fragment spectrum library of metabolites to validate the metabolite identification (secondary annotated metabolites).

The intensity of peak data was further preprocessed by an inhouse software metaX. Those features that were detected in $<50 \%$ of QC samples or $<80 \%$ of biological samples were removed, and the remaining peaks with missing values were imputed with the $\mathrm{k}-\mathrm{NN}$ (k-nearest neighbor) algorithm to further improve the data quality. Principal component analysis (PCA) was performed for outlier detection (outside of $t$ distribution with a $95 \%$ confidence interval) and batch-effects evaluation using the preprocessed dataset. Partial least-squares discriminant analysis (PLS-DA) was done with metaX to discriminate the different variables between groups. $R 2 x, R 2 y$ and $Q 2$ were used to evaluate the fitting effect of the PLS-DA model. $R 2 x$ and $R 2 y$ represent the percentages of $X$ and $Y$ matrix information, respectively, that can be interpreted. The value of $Q 2$ could be acquired through cross-validation calculation. The greater the score of $R 2$ and $Q 2$, the better the prediction effect and the greater the separation degree of the 2 groups of samples in the figure indicating that the classification effect is significant. QC-based robust LOESS signal correction was fitted to the QC data, with respect to the order of injection, to minimize signal intensity drift over time. In addition, the relative standard deviations of the metabolic features were calculated across all QC samples, and those $>30 \%$ were removed.

\section{Western Blot Analysis}

AP exosome proteins were extracted by using radioimmunoprecipitation assay buffer (Suolaibo Biotechnology Co. Ltd., Shanghai, China) supplemented with $1 \%$ phenylmethylsulfonyl fluoride (PMSF) and denatured in $4 \times$ sodium dodecyl sulfonate (SDS) loading buffer. Proteins were then separated on 12\% SDS polyacrylamide gels and transferred by electroblotting onto PVDF membranes (Millipore, Billerica, CA, USA). The PVDF membranes were probed with anti-CD9 antibodies and antitumor susceptibility gene 101 (TSG101) protein antibodies (Bioworld Technology, MN, China) which were exosome markers, and anti- $\beta$ actin antibodies (Abcam, Cambridge, UK) for the control, and then incubated with secondary horseradish peroxidase (HRP)conjugated goat anti-rabbit or anti-mouse antibodies (Jackson ImmunoResearch Laboratories, West Grove, PA, USA). Signals were detected with Immobilon ${ }^{\mathrm{TM}}$ Western chemiluminescent HRP substrate (Millipore).

\section{Transmission Electron Microscopy}

In total, $20 \mu \mathrm{L}$ of a solution of AP exosomes were dropped on the plastic film. The droplet on the plastic film was then covered by a copper mesh. After $10 \mathrm{~min}$ of incubation at room temperature, the copper mesh was dried slowly with a piece of filter paper and followed by a dye process with $20 \mu \mathrm{L}$ of $1 \%$ glutaraldehyde for 5 min. After staining, the copper mesh was dried and then washed 3 times with sterile distilled water. The sample was then dried for 10 min under incandescent light. Finally, the copper mesh was observed and photographed under a transmission electron microscope (JEM-1400, Tokyo, Japan).

\section{Analysis of AP Exosome Proteins}

AP exosome proteins were mainly analyzed by LC-Bio Company in China. Concisely, proteins were first isolated from AP exosomes and then quantified and evaluated through bicinchoninic acid (BCA) and SDS-polyacrylamide gel electrophoresis (PAGE) assay. The qualified proteins were then managed through reductive alkylation and enzymatic hydrolysis. Next, isobaric tags for relative and absolute quantitation (iTRAQ) assay accompanied by high-pH UPLC and LC-MS was performed to analyze the APs exosomal proteins. The Gene Ontology (GO) and KEGG databases were then used to analyze the potential biological functions of the identified proteins.

\section{Cell Lines and Cell Culture}

The chronic myelogenous leukemia cell line K562 and cancer cell lines HepG2 and HCT116 were all purchased from the American Type Culture Collection (ATCC, Manassas, VA, USA). They were cultured in RPMI 1640 medium supplemented with $10 \%$ FBS, $100 \mathrm{U} / \mathrm{mL}$ penicillin, and $100 \mu \mathrm{g} / \mathrm{mL}$ streptomycin (HyClone, Utah, Logan, USA) in a humidified atmosphere of $5 \% \mathrm{CO}_{2}$ at $37^{\circ} \mathrm{C}$.

\section{Cell Viability Assay}

The effects of AP supernatants and exosomes on the viability of K562, HepG2, and HCT116 cell lines were determined by means of cell counting kit (CCK)-8 assay (Dojindo, Kumamoto, Japan). Cancer cells were briefly seeded into 96-well plates at a density of $5 \times 10^{3}$ cells/well with $100 \mu \mathrm{L}$ of complete medium. After being cultured for $24 \mathrm{~h}$ in a humidified incubator with $5 \% \mathrm{CO}_{2}$ at $37^{\circ} \mathrm{C}$, $10 \mu \mathrm{L}$ of AP supernatant or exo-free-AP supernatant or AP exosomes were separately added into experimental wells, while PBS was equally added to the control wells. At indicated time points, 10 $\mu \mathrm{L}$ of CCK-8 solution was added to each well and incubated at $37^{\circ} \mathrm{C}$ for $4 \mathrm{~h}$. Optical density (OD) values were measured at 450 nm using a microplate reader (iMark, Bio-Rad, Hercules, CA, USA). Each experiment was performed in triplicate and repeated 3 times.

\section{Statistical Analysis}

Student's $t$ test was used to detect differences in metabolite concentrations between day- 0 and day- 5 APs. The $p$ value was adjusted for multiple tests using an FDR (Benjamini-Hochberg). The variable important for the projection (VIP) value was calculated, and a VIP cut-off value of 1.0 was used to select important features. Cell viability assay data were analyzed using GraphPad Prism v6.0 (San Diego, CA, USA). Differences between each group were assessed by $t$ tests unless otherwise specified. $p<0.05$ was considered statistically significant.

\section{Results}

\section{Overview of Metabolite Profiling of AP Supernatants}

Accumulating evidence suggests that PSLs can result in various changes. Therefore, we analyzed the metabolite composition of day- 0 and day- 5 stored AP supernatants to understand the process underlying the change in metabolites. As shown in Figure 1A, 11,587 annotated metabolites, including 448 reliable secondary metabolites, were identified from 19,432 totally captured features (in the positive and negative ion modes). We also analyzed the potential pathways in which the annotated metabolites may participate (KEGG pathway analysis). The results indicated that the annotated metabolites were mainly enriched in glycerophospholipid metabolism, choline metabolism in cancer, and metabolic pathways, regardless of whether they were in the positive (Fig. 1B) or negative (Fig. 1C) mode. 
A

\begin{tabular}{cccccc}
\hline Mode & All features & All annotated & MS2 & HMDB & KEGG \\
\hline Positive & 10230 & 6717 & 256 & 5298 & 4056 \\
Negative & 9202 & 4870 & 192 & 3392 & 2736 \\
\hline
\end{tabular}

B

Retrograte endocannabinoid signaling
Regulation of lipolysis in adipocytes
Vitamin digestion and absorption
Fatty digestion and absorption
Arachidonic acid metabolism,
linolenic acid metabolism
Sphingolipid metabolism-

C

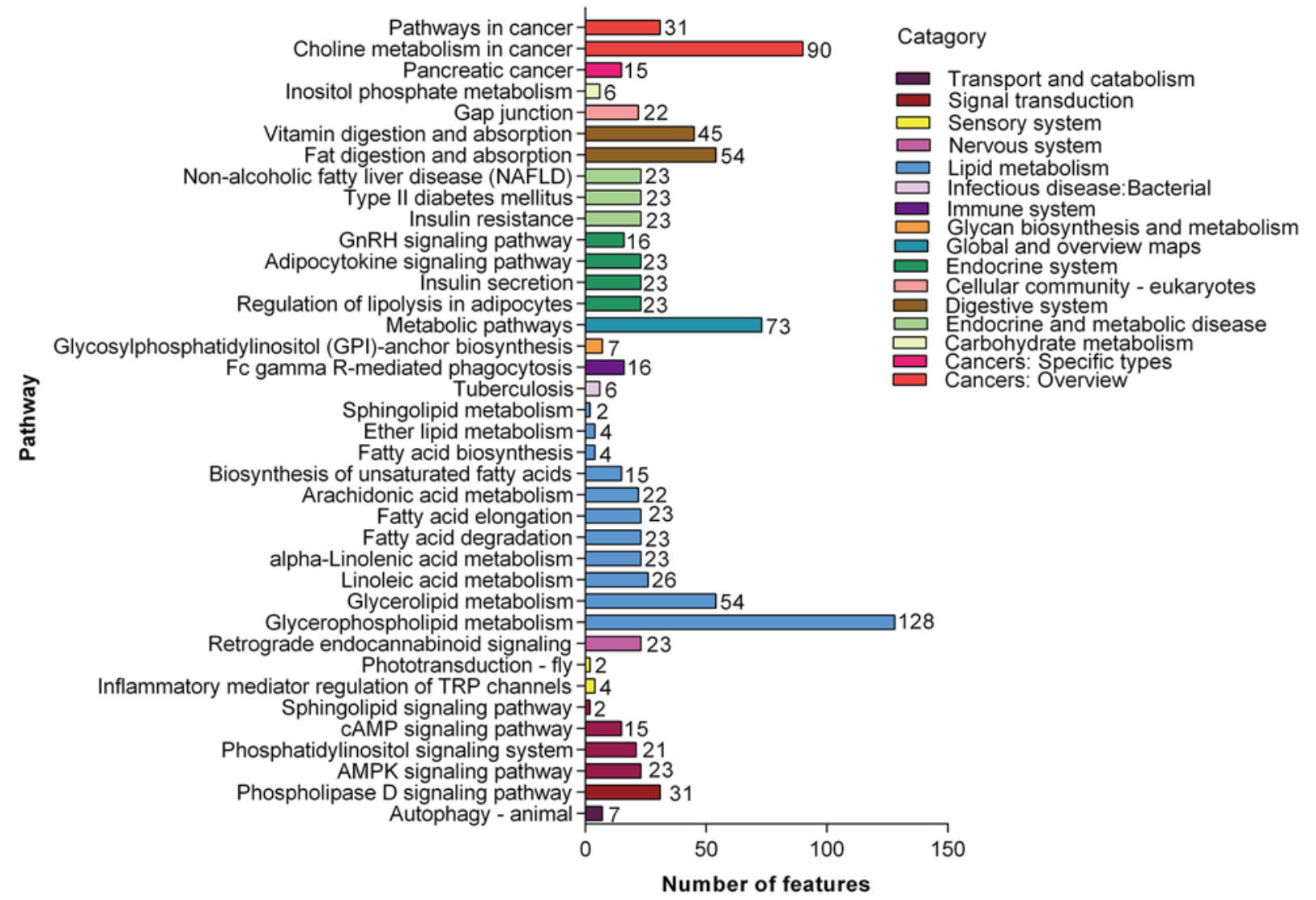

Fig. 1. Identification and KEGG analysis of the annotated metabolites in AP supernatant. A The statistical results of metabolite annotation. A barplot shows the numbers of annotated metabolites in each KEGG pathway in positive (B) and negative $(\mathbf{C})$ (metabolites $>1$ ). 
A

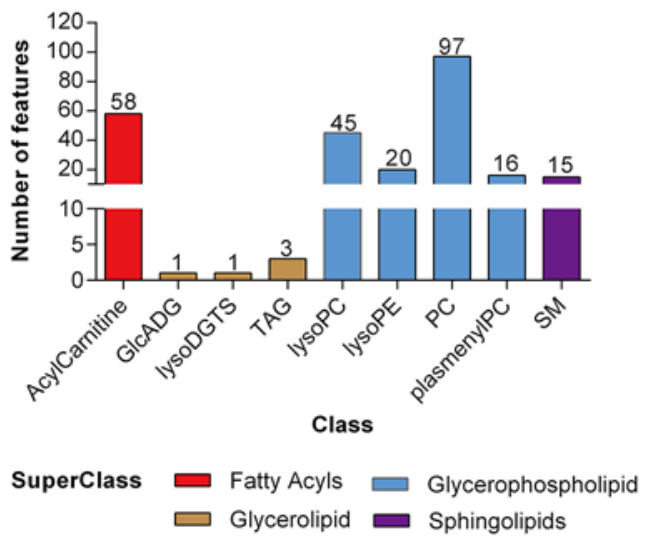

C

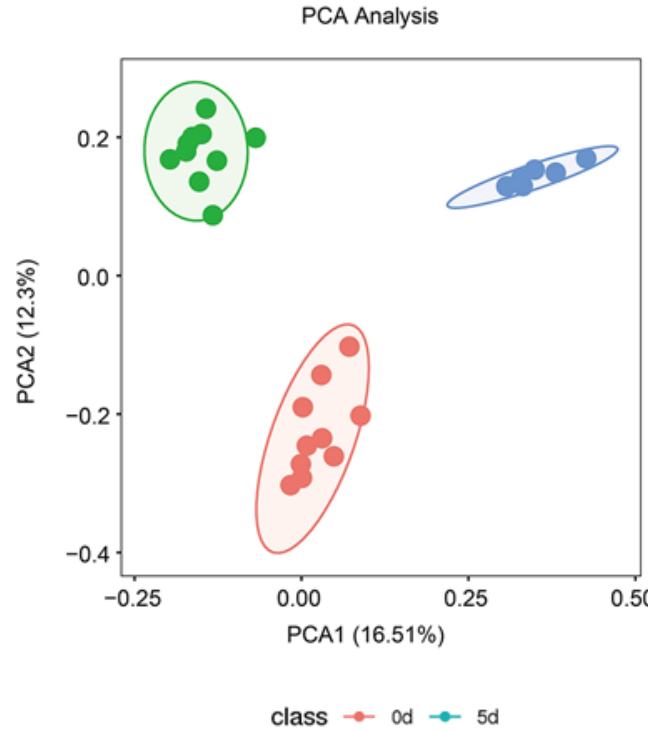

E

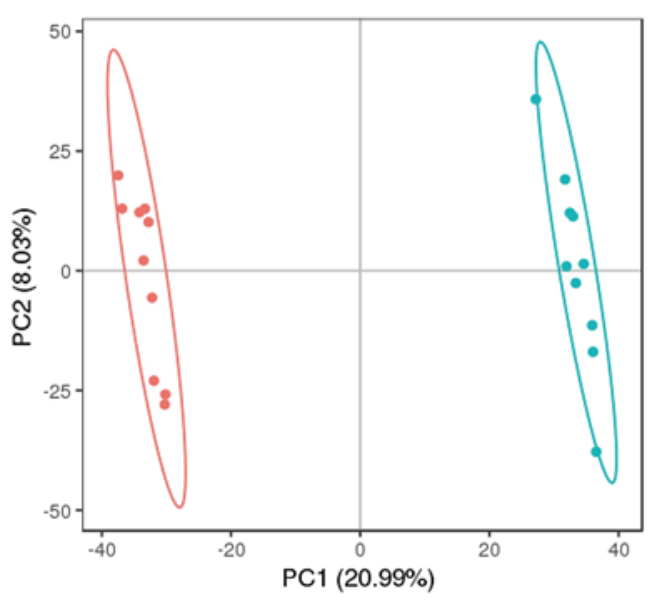

B

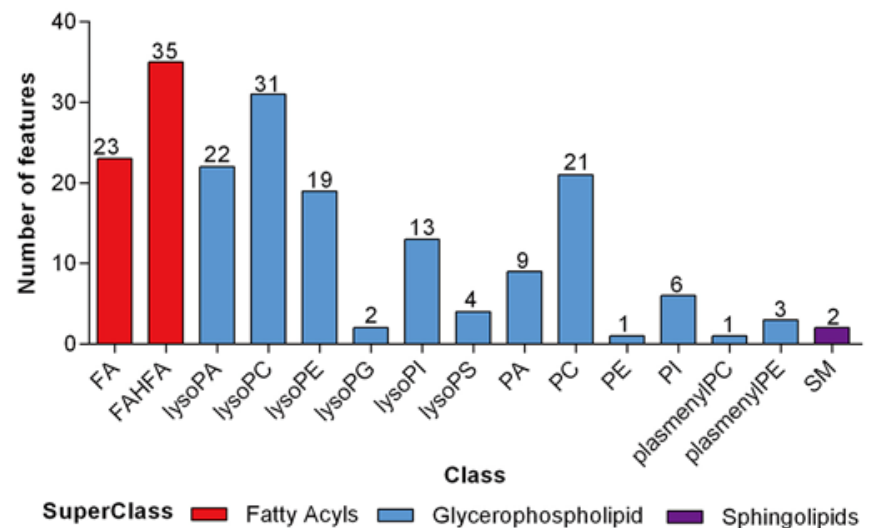

D

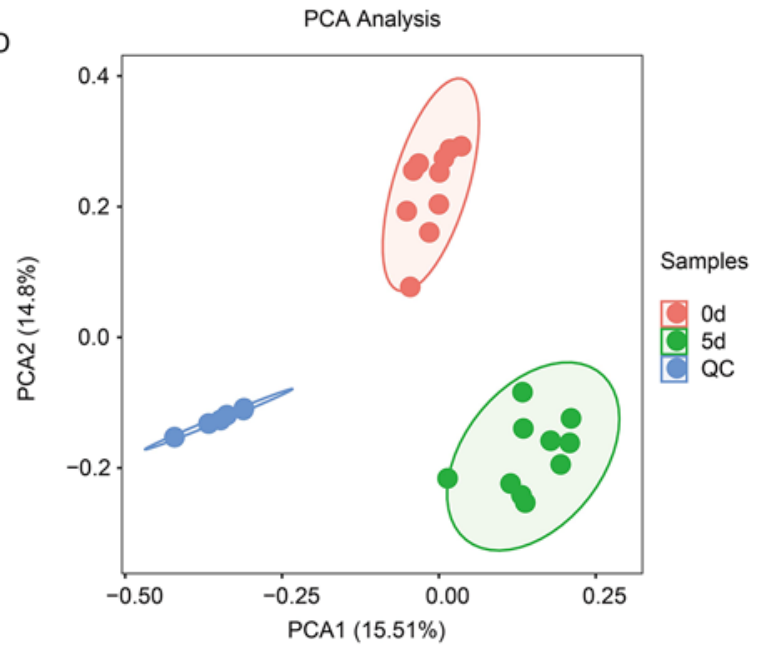

$\mathrm{F}$

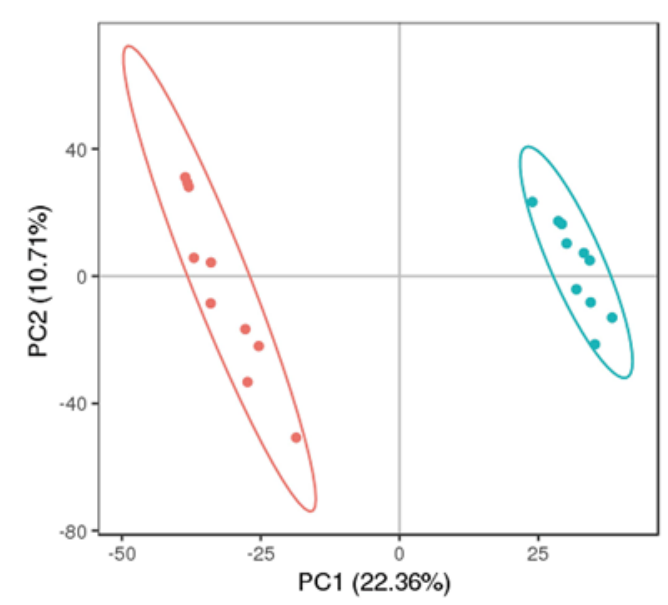

Fig. 2. HMDB classification and PLS-DA show the overview of metabolite profiling of AP supernatant. A, B HMDB classification map of MS2-identified annotated metabolites in the positive (A) and negative (B) mode. PCA shows the similarities and differences between day 0 , day 5 , and the pooled quality control samples in the positive (C) and negative (D) mode. PLS-DA shows the different variables between day- 0 and day- 5 stored AP supernatant groups in the positive (E) and negative (F) mode. 
To further study the 448 reliable secondary metabolites, we performed functional HMDB classification analysis. As displayed in Figure 2 (A, B), the 448 reliable secondary metabolites were mainly classified into fatty acyls, glycerophospholipids, and sphingolipids. In addition, PCA showed that the metabolites had a high degree of similarity in day- 0 , day- 5 , and control samples, and that the principal components in the day- 0 and day- 5 stored AP supernatant metabolites were different in both the positive (Fig. 2C) and negative (Fig. 2D) mode. To further address whether the metabolites in AP supernatants changed during storage, PLS-DA was used to discern the different variables in the day- 0 and day- 5 groups. The results showed that the cumulative $R 2$ and $Q 2$ values were all $>0.92$ in both the positive (Fig. 2E) and negative (Fig. 2F) mode, indicating that the day- 0 and day- 5 stored AP supernatants exhibited significantly different metabolites. All these data demonstrate that AP metabolism profiling significantly changes during storage.

\section{Differential Supernatant Metabolite Analysis in Day-0 and Day-5 Stored APs}

These results prompted us to investigate how the metabolites in AP supernatants changed. We analyzed the differences detected in the supernatant metabolites of day- 0 and day- 5 stored APs. The heatmap revealed that day- 0 and day- 5 stored AP supernatants had significantly different metabolite profiling regardless of whether they were in the positive (Fig. 3A) or negative (Fig. 3B) mode. In the differential metabolite analysis (fold change [FC] $\geq 2$ or FC $\leq 1 / 2, q$ value $[t$ test] $p \leq 0.05$ [BenjaminiHochberg-corrected], VIP $\leq 1), 297$ metabolites were found to be upregulated and 434 downregulated in the day- 5 stored AP supernatants compared to the day- 0 ones in the positive mode. Similar results were found in the negative mode accompanied by 370 upregulations and 229 downregulations of metabolites in the day- 5 AP supernatants. To further investigate the potential function of differential metabolites, KEGG pathway analysis was performed in both the positive and negative mode. As shown in Figure 3C (positive mode) and Figure 3D (negative mode), the upregulated metabolites in the day- 5 stored AP supernatants participated significantly in the pathways of biosynthesis of unsaturated fatty acids and linolenic acid metabolism. Interestingly, we discovered that many upregulated metabolites such as retinoate and 9-cis-retinoic acid are involved in cancer-related pathways, e.g., those in nonsmall-cell lung cancer and small-cell lung cancer. This reminded us that the terminal-stored APs may participate in the regulation of cancer. In addition, the downregulated metabolites identified in the positive and negative mode participated mainly in the pathways of arachidonic acid metabolism, serotonergic synapse, linolenic acid metabolism, and bile secretion (Fig. 3E, F).

\section{Identification and Classification of Proteins in AP} Exosomes

To further understand the changes occurring in APs during storage, AP exosomes from 6 healthy blood donors were also extracted from day- 0 and day- 5 stored APs supernatants by ultracentrifugation (Fig. 4A), and then mixed to form an AP exosome pool. The exosome markers CD9 and TSG101 were then tested. As shown in Figure 4B, CD9 and TSG101 were successfully detected, suggesting that AP exosomes were successfully isolated. Meanwhile, the morphology of AP exosomes was evaluated by TEM. The results showed that AP exosomes in the isolated fractions were oval or bowl-shaped (Fig. 4C). Furthermore, the amount and volume of AP exosomes on day 5 were greater than on day 0 , reflecting that they also changed during storage.

To identify proteins in AP exosomes during storage, iTRAQ quantification was performed in pooled AP exosomes ( 6 randomly selected day- 0 or day- 5 AP exosomes). In total, 607 proteins were identified. At the same time, the results of the KEGG pathway analysis showed that these proteins chiefly participated in the pathways of transport and catabolism, the immune system, and cancers (Fig. 4D).

\section{Analysis of the Protein Change in AP Exosomes during Storage}

To analyze the protein changes in AP exosomes during storage, we compared day- 0 and day- 5 AP exosome proteins. The heatmap showing the top 20 exosome proteins that were significantly different on day 0 and day 5 indicated that the day- 0 and day- 5 AP exosomes had significantly different protein profiling (Fig. 5A). Day-5 stored AP exosomes exhibited 292 upregulated and 53 downregulated proteins compared with the day- 0 stored $\mathrm{AP}$ exosomes $(p<0.05, \mathrm{FC}<0.83$ or $\mathrm{FC}>1.2)$. We then performed GO-enrichment analysis to analyze the main function of the differential proteins in the day- 0 and day5 stored AP exosomes. The results were that the upregulated proteins in the day-5 AP exosomes, i.e., which mainly belonged to the cellular component of the cell junction, extracellular region, and cellular membrane, had the molecular functions of binding, catalytic activity, molecular function regulation, and structural molecule activity; they may play a significant role in biological adhesion, metabolism, and the multicellular process of the organism (Fig. 5B). However, as shown in Figure 5C, the downregulated proteins in the day- 5 AP exosomes, i.e., mainly extracellular region and protein-containing complexes, may perform the molecule activity of binding and transporter activity, and participate in the multicellular process of the organism.

To further investigate in which pathways the aberrantly regulated proteins were involved, we carried out the
Li/Zhang/Cheng/Yu/Wang 


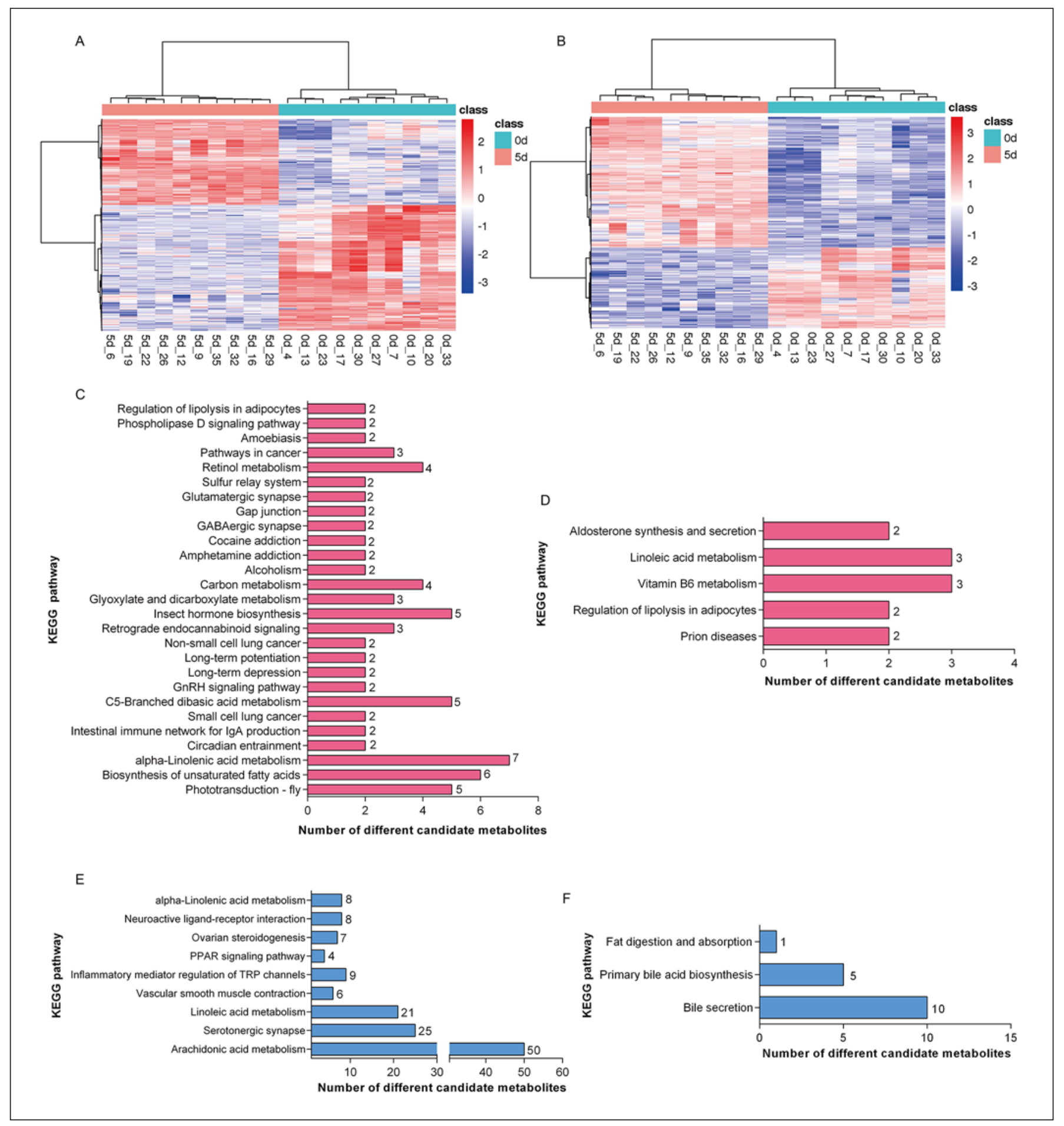

Fig. 3. Differential analysis of metabolites in the AP supernatant. Heatmap shows the metabolite difference annotated in the positive (A) and negative (B) mode between day- 0 and day-5 AP supernatant. KEGG analysis of the upregulated metabolites: positive mode-annotated (C) and negative mode-annotated (D) enriched pathways. KEGG analysis of the downregulated metabolites: positive mode-annotated (C) and negative mode-annotated (D) enriched pathways.
KEGG pathway analysis. As shown in Figure 5D, the upregulated proteins participated mainly in the pathways of systemic lupus erythematosus, viral carcinogenesis, Staphylococcus aureus infection, platelet activation, and the ras-related protein (Rap1) signaling pathway. We could also decipher that the downregulated proteins were primarily involved in transcriptional misregulation in cancer and cholesterol metabolism (Fig. 5E). 


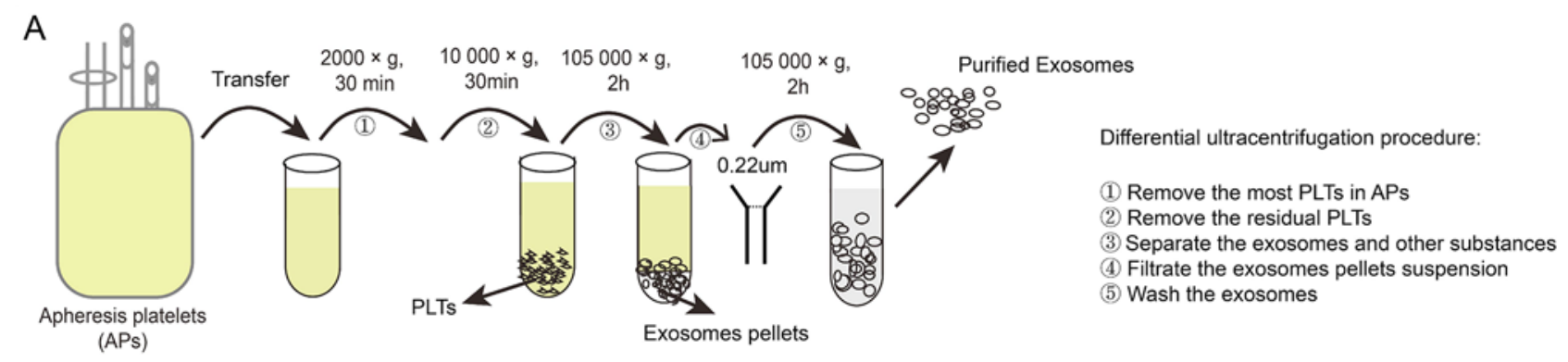

B

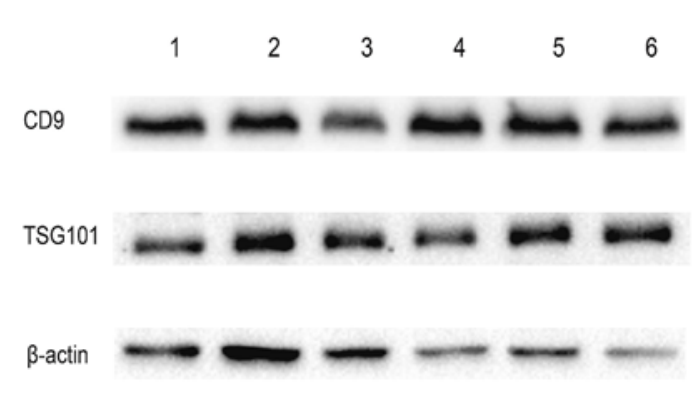

C

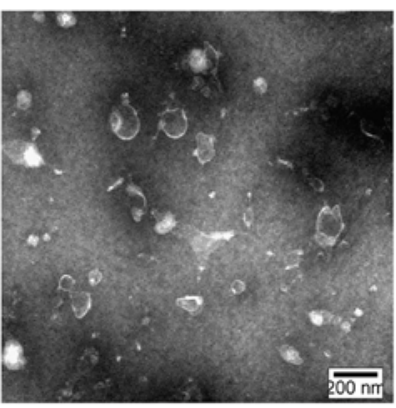

Day 0

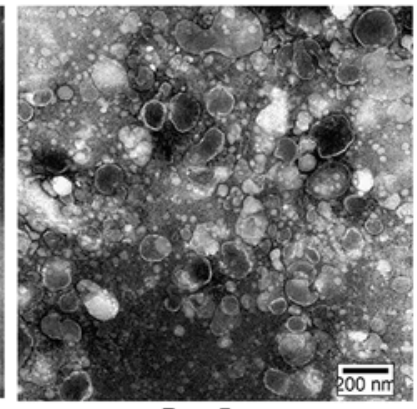

Day 5

D
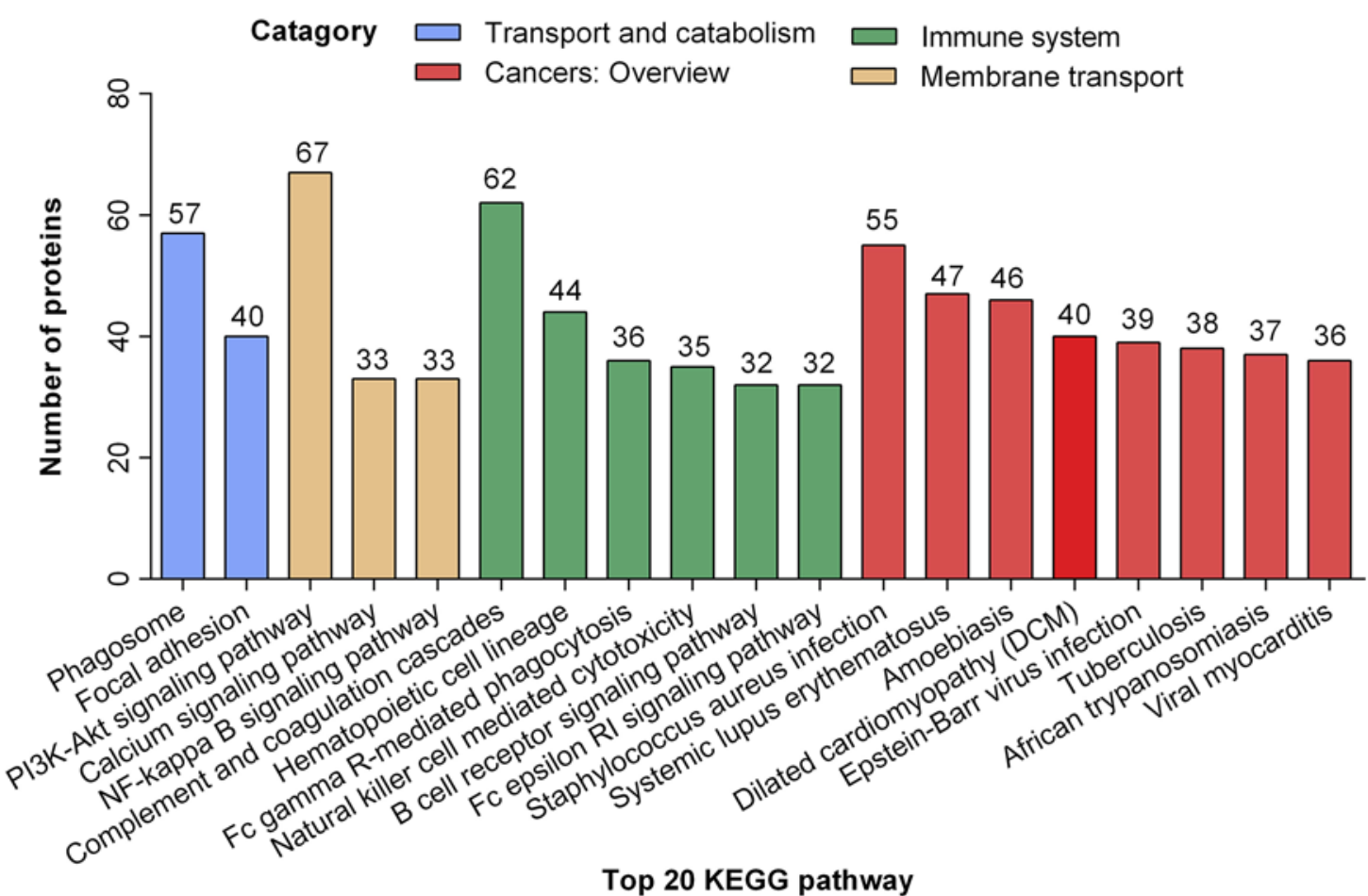

Top 20 KEGG pathway

Fig. 4. A Isolation (by multistep centrifugation) and classification of AP exosomes. B The expression of exosome marker was analyzed by Western blot assay. C Transmission electron microscopy shows the morphology difference between day 0 and day 5. D KEGG analysis shows the top 20 pathways in which iTRAQ-identifiable proteins were enriched. 


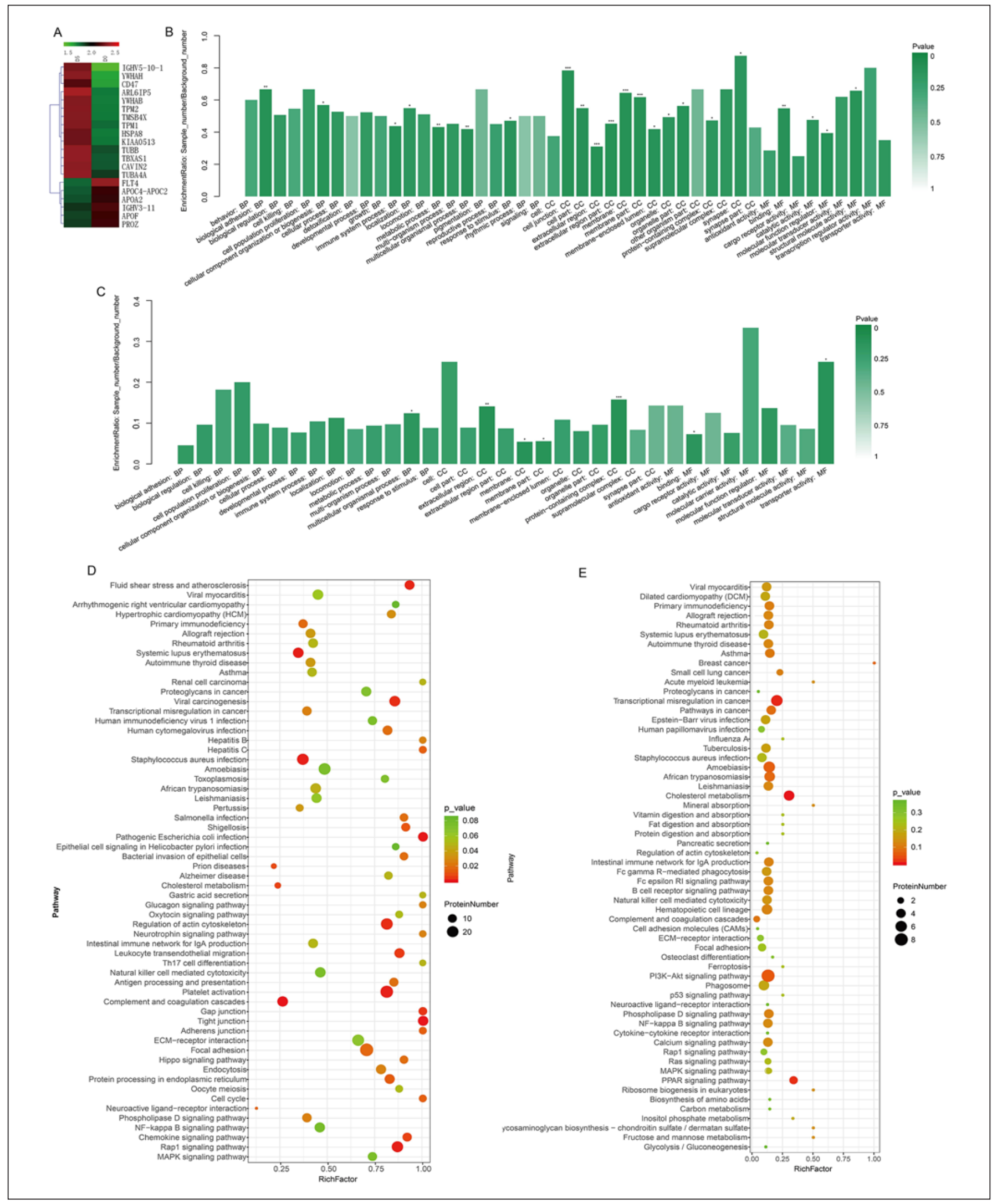

Fig. 5. Differential protein analysis between day-0 and day-5 AP exosomes. A Heatmap shows the protein difference between day- 0 and day-5 AP exosomes. B A barplot of GO-enrichment analysis (biological process, cellular component, and molecular function) of the upregulated proteins in day-5 AP exosomes. C A barplot of
GO-enrichment analysis (biological process, cellular component, and molecular function) of the downregulated proteins in day- 5 AP exosomes. D, E A bubble plot of the enriched KEGG pathways of upregulated (D) or downregulated (E) proteins in day-5 AP exosomes. 


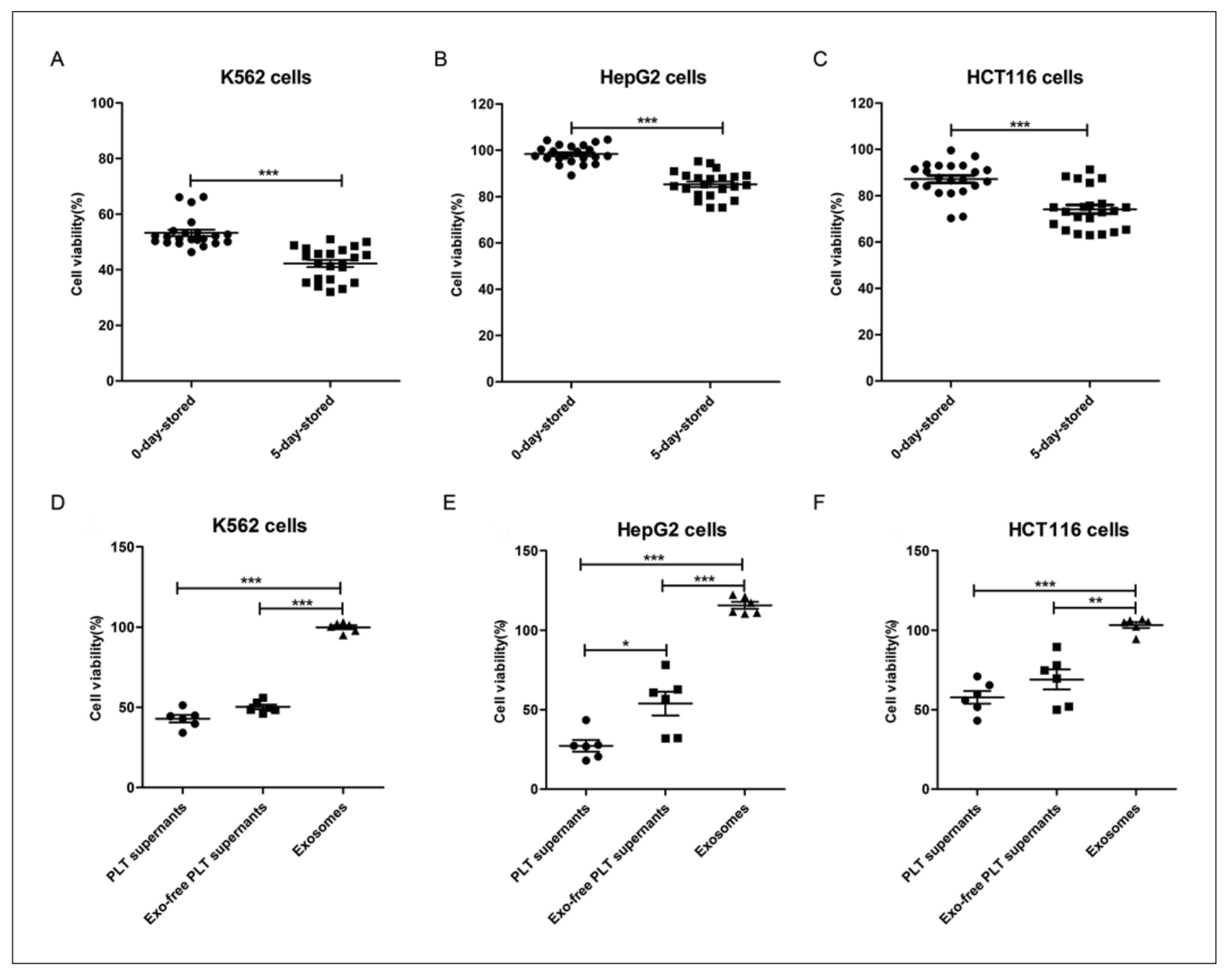

Fig. 6. The effects of terminal-stored AP supernatants and exosomes on the proliferation of cancer cells. A scatter plot shows the inhibition of day- 5 stored AP supernatant on the proliferation of K562 (A), HepG2 (B), and HCT116 (C) cancer cells; the data are shown as mean \pm SD of 3 independent experiments and statistical significance of the results was calculated by Student's $t$ test.
*** $p<0.001$. A scatter plot shows the inhibition of day- 5 stored AP supernatant, exosome-free AP supernatant, and AP exosomes on the proliferation of K562 (D), HepG2 (E), and HCT116 (F) cancer cells; the data are shown as mean \pm SD of 3 independent experiments and statistical significance of the results was calculated by Student's $t$ test. ${ }^{* * *} p<0.001$; $^{* *} p<0.01$; $^{*} p<0.05$.
The Influence of Day-5 Terminal-Stored AP Supernatants and Exosomes on the Proliferation of Cancer Cells

Our analysis of metabolomics and proteomics in AP supernatants and exosomes indicated that multiple metabolites and proteins are involved in cancer-related pathways. We assessed the effects of AP supernatants and exosomes on the proliferation of K562, HepG2, and HCT116 cell lines by means of CCK- 8 assays. As shown in Figure 6A-C, day- 5 stored AP supernatants could restrain the proliferation ability of K562, HepG2, and HCT116 cells more effectively than day-0 stored AP supernatants. However, the day- 5 stored AP exosomes had little effect on the proliferation capability of K562, HepG2, and HCT116 cancer cells (Fig. 6E, F).

\section{Discussion}

APs play an irreplaceable role in the prevention and treatment of bleeding in patients with thrombocytopenia. Furthermore, accumulating evidence suggests that APs, which may develop PSLs during storage [14, 15], contribute to the regulation of the immune system and cancer progression [16-21]. This facilitates a profound understanding of the characteristics of APs. It is thus essential 
to explore the changes that occur in substances in APs during storage.

Given that day 5 is the terminal time of AP storage according to Chinese blood storage requirements, we selected a timeline consisting of day- 0 and day- 5 stored APs as our object of study. We were able to confirm that the day- 0 and day- 5 stored APs showed significantly different metabolomics in their supernatants, and found that the upregulated metabolites retinoate and 9-cis-retinoic acid in day- 5 stored AP supernatants were enriched in cancer-related KEGG pathways. There are also studies that indicate that retinoate and 9-cis-retinoic acid are inversely related to malignant features [22-25]. Some reports state that retinoate and 9-cis-retinoic acid both participate in the regulation of the immune system. There are studies that report that retinoate is involved in the induction of regulatory $\mathrm{T}$ cells and the inhibition of the differentiation of naïve T cells into Th17 cells [26-28], suggesting that retinoate plays an important role in balancing the immune system. Besides, 9-cis-retinoic acid can induce a distinct regulatory dendritic cell phenotype that inhibits murine delayed-type allergy, and it can inhibit the inflammatory response of adherent monocytes and increase their ability to induce classical monocyte migration $[29,30]$, indicating that it may inhibit the immune response. All these data remind us that the day-5 terminal-stored AP supernatants may regulate the malignant features of cancer and the immune response. Our results validated the notion that the terminal-stored AP supernatants inhibit the proliferation of cancer cells. Our results confirm that APs undergo an alteration of metabolites during storage. We do, however, believe that the terminal-stored APs may be more beneficial to cancer patients and patients with immune-mediated thrombocytopenia. This requires further study.

There are many studies on the contents of platelet microparticles (PMPs) [31-33], but only a few have investigated the variation of proteins in AP exosomes. We performed iTRAQ proteomics to identify the different proteins in day- 0 and day- 5 stored AP exosomes, and found that the day-5 stored AP exosomes had 292 upregulated and 53 downregulated proteins. By KEGG analysis, we discovered that many upregulated proteins such as 14-33 protein $\eta$ (YWHAH), $14-3-3$ protein $\beta / \alpha$ (YWHAB), and transforming growth factor $\beta 1$ proprotein (TGFB1) as well as downregulated proteins such as insulin-like growth factor-binding protein 3 (IGFBP3) and macrophage colony-stimulating factor 1 receptor (CSF1R) in day-5 stored AP exosomes were enriched in cancer-related pathways like viral carcinogenesis, transcriptional misregulation in cancer, and renal cell carcinoma. Many authors have indicated that YWHAH, YWHAB, and TGFB1 are positively correlated with cancer malignant features [34-36], while IGFBP3 and CSF1R are negative- ly correlated with poor outcomes [37, 38]. Our data indicated that the day- 5 stored AP exosomes had no significant impact on the proliferation of cancer cells, which may attribute to the balance between the cancer promotion- and inhibition-related proteins in AP exosomes.

In summary, our study documents the changes that occur in AP supernatant metabolomics and AP exosome proteomics. Furthermore, terminal AP supernatants may contribute to inhibiting the proliferation of cancer cells and are therefore possibly better for use in cancer patients. However, further study is required to explore the mechanism of AP supernatant metabolites involved in inhibiting cancer cell proliferation. Many differential metabolites and exosome proteins in freshly prepared and terminal-stored APs may be involved in the regulation of inflammation, reminding us to pay attention to the transfusion of APs in patients with inflammatory diseases. This warrants further study. Nevertheless, these findings enhance our understanding of the variation of substances in APs during storage and their effects on cancer cells, which can contribute to the better clinical use of APs.

\section{Statement of Ethics}

All donors gave their written informed consent and this study was approved by the ethics committee of the Chinese People's Liberation Army (PLA) General Hospital.

\section{Conflict of Interest Statement}

All authors declare no conflicts of interest.

\section{Funding Sources}

This work was supported by the National Natural Science Foundation of China (grant No. 81770194) and the Digestive Medical Coordinated Development Center of Beijing Hospital Authority (grant No. XXX0108).

\section{Author Contributions}

X.L. performed most of the experiments. Y.Z. analyzed the experimental data and draw up the manuscript. F.C. and Y.Y. provided experimental and technical assistance in the acquisition of data. D.W. designed and supervised the study, and was a major contributor in modifying the manuscript. All authors read and approved the final version. 


\section{References}

1 Elemary M, Seghatchian J, Stakiw J, Bosch M, Sabry W, Goubran H. Transfusion challenges in hematology oncology and hematopoietic stem cell transplant - Literature review and local experience. Transfus Apheresis Sci. 2017 Jun;56(3):317-21.

2 Pitman JP, Basavaraju SV, Shiraishi RW, Wilkinson R, von Finckenstein B, Lowrance DW, et al. Namibia's transition from whole blood-derived pooled platelets to single-donor apheresis platelet collections. Transfusion. 2015 Jul;55(7):1685-92.

3 Kamhieh-Milz J, Mustafa SA, Sterzer V, Celik H, Keski S, Khorramshahi O, et al. Secretome profiling of apheresis platelet supernatants during routine storage via antibody-based microarray. J Proteomics. 2017 Jan;150:7485.

4 Kreuger AL, Rostgaard K, Middelburg RA, Kerkhoffs JH, Edgren G, Erikstrup C, et al. Storage time of platelet concentrates and risk of a positive blood culture: a nationwide cohort study. Transfusion. 2018 Jan;58(1):1624.

5 Klinger MH. The storage lesion of platelets: ultrastructural and functional aspects. Ann Hematol. 1996 Sep;73(3):103-12.

6 Tkach M, Théry C. Communication by Extracellular Vesicles: Where We Are and Where We Need to Go. Cell. 2016 Mar;164(6):122632.

7 Tao SC, Guo SC, Zhang CQ. Platelet-derived Extracellular Vesicles: An Emerging Therapeutic Approach. Int J Biol Sci. 2017 Jul;13(7): 828-34.

8 Sadallah S, Schmied L, Eken C, Charoudeh HN, Amicarella F, Schifferli JA. Platelet-Derived Ectosomes Reduce NK Cell Function. J Immunol. 2016 Sep;197(5):1663-71.

9 Dinkla S, van Cranenbroek B, van der Heijden WA, He X, Wallbrecher R, Dumitriu IE, et al. Platelet microparticles inhibit IL-17 production by regulatory $\mathrm{T}$ cells through $\mathrm{P}$-selectin. Blood. 2016 Apr;127(16):1976-86.

10 Goubran H, Sabry W, Kotb R, Seghatchian J, Burnouf T. Platelet microparticles and cancer: an intimate cross-talk. Transfus Apheresis Sci. 2015 Oct;53(2):168-72.

11 Goubran HA, Elemary M, Radosevich M, Seghatchian J, El-Ekiaby M, Burnouf T. Impact of Transfusion on Cancer Growth and Outcome. Cancer Growth Metastasis. 2016 Mar;9:1-8.

12 Pienimaeki-Roemer A, Konovalova T, Musri MM, Sigruener A, Boettcher A, Meister G, et al. Transcriptomic profiling of platelet senescence and platelet extracellular vesicles. Transfusion. 2017 Jan;57(1):144-56.

13 Wang S, Jiang T, Fan Y, Zhao S. A proteomic approach reveals the variation in human platelet protein composition after storage at different temperatures. Platelets. 2019;30(3): 403-12.
14 Handigund M, Cho YG. Insights into Platelet Storage and the Need for Multiple Approaches. Ann Clin Lab Sci. 2015;45(6):713-9.

15 Södergren AL, Tynngård N, Berlin G, Ramström S. Responsiveness of platelets during storage studied with flow cytometry-formation of platelet subpopulations and LAMP1 as new markers for the platelet storage lesion. Vox Sang. 2016 Feb;110(2):116-25.

16 Rondina MT, Garraud O. Emerging evidence for platelets as immune and inflammatory effector cells. Front Immunol. 2014 Dec;5:653.

17 Rondina MT, Weyrich AS, Zimmerman GA. Platelets as cellular effectors of inflammation in vascular diseases. Circ Res. 2013 May; 112(11):1506-19.

18 Semple JW, Italiano JE Jr, Freedman J. Platelets and the immune continuum. Nat Rev Immunol. 2011 Apr;11(4):264-74.

19 Kuznetsov HS, Marsh T, Markens BA, Castaño Z, Greene-Colozzi A, Hay SA, et al. Identification of luminal breast cancers that establish a tumor-supportive macroenvironment defined by proangiogenic platelets and bone marrow-derived cells. Cancer Discov. 2012 Dec;2(12):1150-65.

20 Wojtukiewicz MZ, Sierko E, Hempel D, Tucker SC, Honn KV. Platelets and cancer angiogenesis nexus. Cancer Metastasis Rev. 2017 Jun;36(2):249-62.

21 Ng MS, Tung JP, Fraser JF. Platelet Storage Lesions: What More Do We Know Now? Transfus Med Rev. 2018 Apr;32(3):S08877963(17)30189-X

22 Bama ES, Grace VMB, Sundaram V, Jesubatham PD. Synergistic effect of co-treatment with all-trans retinoic acid and 9-cis retinoic acid on human lung cancer cell line at molecular level. 3 Biotech. 2019;9(4):159.

$23 \mathrm{Wu}$, Yang R, Zhang L, Li Y, Liu B, Kang H, et al. Metabolomics research on potential role for 9-cis-retinoic acid in breast cancer progression. Cancer Sci. 2018 Jul;109(7):231526.

24 Eskra JN, Kuiper JW, Walden PD, Bosland MC, Özten N. Interactive effects of 9-cis-retinoic acid and androgen on proliferation, differentiation, and apoptosis of LNCaP prostate cancer cells. Eur J Cancer Prev. 2017 Jan; 26(1):71-7.

25 Shi B, Li LM, You SQ, Xu SP, Han R. [Inhibition of tumor invasion and metastasis by retinoid 4-APR]. Yao Xue Xue Bao. 1997;32(1): 33-7.

26 Bettelli E, Carrier Y, Gao W, Korn T, Strom TB, Oukka M, et al. Reciprocal developmental pathways for the generation of pathogenic effector TH17 and regulatory T cells. Nature. 2006 May;441(7090):235-8.
27 Xiao S, Jin H, Korn T, Liu SM, Oukka M, Lim B, et al. Retinoic acid increases Foxp3+ regulatory $\mathrm{T}$ cells and inhibits development of Th17 cells by enhancing TGF-beta-driven Smad3 signaling and inhibiting IL-6 and IL23 receptor expression. J Immunol. 2008 Aug; 181(4):2277-84.

28 Hill JA, Hall JA, Sun CM, Cai Q, Ghyselinck $\mathrm{N}$, Chambon $\mathrm{P}$, et al. Retinoic acid enhances Foxp 3 induction indirectly by relieving inhibition from CD4+CD44hi Cells. Immunity. 2008 Nov;29(5):758-70.

29 Kraus LF, Scheurmann N, Frenzel DF, Tasdogan A, Weiss JM. 9-cis-Retinoic acid induces a distinct regulatory dendritic cell phenotype that modulates murine delayed-type allergy. Contact Dermat. 2018 Jan;78(1):41-54.

$30 \mathrm{Xu}$ J, Drew PD. 9-Cis-retinoic acid suppresses inflammatory responses of microglia and astrocytes. J Neuroimmunol. 2006 Feb;171(12):135-44.

31 Michael JV, Wurtzel JG, Mao GF, Rao AK, Kolpakov MA, Sabri A, et al. Platelet microparticles infiltrating solid tumors transfer miRNAs that suppress tumor growth. Blood. 2017 Aug; 130(5):567-80.

32 Liang H, Yan X, Pan Y, Wang Y, Wang N, Li L, et al. MicroRNA-223 delivered by plateletderived microvesicles promotes lung cancer cell invasion via targeting tumor suppressor EPB41L3. Mol Cancer. 2015 Mar;14(1):58.

33 Laffont B, Corduan A, Plé H, Duchez AC, Cloutier N, Boilard E, et al. Activated platelets can deliver mRNA regulatory Ago $2 \cdot$ microRNA complexes to endothelial cells via microparticles. Blood. 2013 Jul;122(2):253-61.

34 Kaneko S, Matsumoto K, Minamida S, Hirayama $\mathrm{T}$, Fujita $\mathrm{T}$, Kodera $\mathrm{Y}$, et al. Incremental Expression of 14-3-3 Protein beta/alpha in Urine Correlates with Advanced Stage and Poor Survival in Patients with Clear Cell Renal Cell Carcinoma. Asian Pac J Cancer Prev. 2016;17(3):1399-404.

35 Park GY, Han JY, Han YK, Kim SD, Kim JS, Jo WS, et al. 14-3-3 eta depletion sensitizes glioblastoma cells to irradiation due to enhanced mitotic cell death. Cancer Gene Ther. 2014 Apr;21(4):158-63.

36 Leitlein J, Aulwurm S, Waltereit R, Naumann U, Wagenknecht B, Garten W, et al. Processing of immunosuppressive pro-TGF-beta 1,2 by human glioblastoma cells involves cytoplasmic and secreted furin-like proteases. Immunol. 2001 Jun;166(12):7238-43.

37 Kluger HM, Dolled-Filhart M, Rodov S, Kacinski BM, Camp RL, Rimm DL. Macrophage colony-stimulating factor-1 receptor expression is associated with poor outcome in breast cancer by large cohort tissue microarray analysis. Clin Cancer Res. 2004 Jan;10(1 Pt 1) 173-7.

38 Yang L, Li J, Fu S, Ren P, Tang J, Wang N, et al. Up-regulation of Insulin-like Growth Factor Binding Protein-3 Is Associated with Brain Metastasis in Lung Adenocarcinoma. Mol Cells. 2019 Apr;42(4):321-32. 